\title{
Grónica general de la Legislatura Post-Bipartidista
}

$$
\text { Victor F. Vázquez Alonso }
$$

La X legislatura ha certificado el fin de un sistema político en Andalucía que, como en la gran mayoría de los subsistemas autonómicos, estaba caracterizado por la propia lógica bipartidista, y en buena medida también, semi-presidencialista, que hasta hace muy poco regía en el parlamentarismo español a nivel nacional. Prueba de ello fueron los 81 días que hubieron de transcurrir tras la celebración de las elecciones, para que la candidata del PSOE obtuviera la confianza de la Cámara con los votos del su grupo parlamentario y el de Ciudadanos: un total de 56 síes frente a 51 noes. Sin duda alguna, pese a que esa ruptura con el paradigma bipartidista no significó un cambio en el partido de gobierno andaluz, sí que ha tenido una incidencia relevante en las instituciones parlamentarias de la comunidad autónoma, habiéndose dado, en este sentido, circunstancias hasta ahora inéditas desde el origen de la autonomía andaluza. A este respecto, lo primero que se podría señalar es que durante a X legislatura han adquirido un especial protagonismo los mecanismos de control parlamentario. Así, más allá de que, como bien ponen de manifiesto las cifras ${ }^{1}$, han sido muchas las iniciativas de control a las que ha tenido que hacer frente el gobierno, cabe destacar especialmente que se han llevado a cabo hasta tres comisiones de investigación de especial significado político, como han sido: la "Comisión de investigación relativa a la gestión de los fondos públicos, las encomiendas de gestión y subvenciones concedidas a la Fundación Andaluza Fondo de Formación y Empleo (FAFFE) desde su creación hasta la actualidad"; la "Comisión de Investigación relativa a las concesiones de las subvenciones otorgadas por la administración de la Junta de Andalucía en materia de Formación Profesional para el Empleo (FPE), desde 2002 hasta la actualidad, incluida su gestión, evaluación y justificación"; y la "Comisión de investigación relativa a un posible uso irregular de tarjetas de la extinta Fundación Andaluza de Formación y Empleo (FAFFE), siendo las dos primeras propuestas por el grupo parlamentario popular y la última a propuesta de ciudadanos.

En cualquier caso, puede subrayarse que, a pesar de la fragmentación del sistema de partidos en el Parlamento andaluz, y de la propia precariedad de la mayoría de gobierno, la X legislatura, como se resumirá continuación, no se ha caracterizado por la poca actividad legislativa, sino más bien al contrario. Todo ello sin obviar que ha habido ámbitos en los que se ha estado lejos de cumplir con las expectativas normativas proyectadas desde el gobierno andaluz, entre los que destacan el ámbito

$1 \quad$ Así, durante la X legislatura se han llevado a cabo 177 Interpelaciones; 6753 preguntas escritas; 2688 preguntas orales en pleno y 6788 preguntas orales en comisión. 
agrícola, y también el relacionado con la infancia, sobresaliendo aquí la cuestión aun no resuelta en sede parlamentaria, de la bio-climatización de las aulas. Concretamente, se han aprobado un total de 31 leyes, cuya procedencia ha sido en 24 ocasiones un proyecto de ley, siendo el resto propuestas de ley, en la mayoría de los casos suscritas por varios grupos parlamentarios. Igualmente, durante esta legislatura se ha aprobado un decreto legislativo, como es sabido, el 1/2018, de 19 de junio, por el que se aprueba el texto refundido de las disposiciones dictadas por la Comunidad Autónoma de Andalucía en materia de tributos cedidos.

Tomando en consideración el contenido de estas leyes, podríamos decir, en primer lugar, que bien puede calificarse la X legislatura como una legislatura de marcado carácter social. Como puede verse a continuación, buena parte de los trabajos legislativos bien pueden situarse en el eje de políticas públicas en materia de igualdad y extensión de derechos:

Ley de modificación de la Ley 12/2007, de 26 de noviembre, para la promoción de la igualdad de género en Andalucía

Ley por la que se modifica la Ley 13/2007, de 26 de noviembre, de medidas de prevención y protección integral contra la violencia de género

Ley Andaluza del Voluntariado

Ley de los Derechos y la Atención a las Personas con Discapacidad en Andalucía

Ley de Servicios Sociales de Andalucía

Ley de medidas urgentes para favorecer la inserción laboral, la estabilidad en el empleo, el retorno del talento y el fomento del trabajo autónomo (procedente del Decreto-Ley 2/2015, de 3 de marzo)

Ley para garantizar los derechos, la igualdad de trato y no discriminación de las personas LGTBI y sus familiares en Andalucía.

La X legislatura se inserta igualmente en un contexto de recuperación y reacción frente a la crisis económica en España. A este respecto, podríamos destacar determinadas iniciativas en materia de función pública y empleo como las siguientes:

Ley relativa a medidas extraordinarias para el establecimiento de ayudas a los trabajadores afectados por el expediente de regulación de empleo $\mathrm{n}^{\circ}$ 160/2004, correspondiente a la empresa Hitemasa

Ley de modificación de la Ley 3/2005, de 8 de abril, de Incompatibilidades de Altos Cargos de la Administración de la Junta de Andalucía y de 
Declaración de Actividades, Bienes, Intereses y Retribuciones de Altos Cargos y otros Cargos Públicos

Ley por la que se modifica la Ley 6/1985, de 28 de noviembre, de Ordenación de la Función Pública de la Junta de Andalucía, en materia de valoración de la antigüedad a efectos de méritos

Ley de medidas urgentes para favorecer la inserción laboral, la estabilidad en el empleo, el retorno del talento y el fomento del trabajo autónomo (procedente del Decreto-Ley 2/2015, de 3 de marzo)

A estas medidas habría que sumar algunos de los Decretos Leyes aprobados bajo el presupuesto habilitante de la "coyuntura económica problemática", a través de los cuales, como vemos, se articulan igualmente políticas de reacción frente a ciertos aspectos sociales o puramente económicos de la crisis:

Decreto Ley 4/2015, de 27 de agosto, por el que se modifican determinados artículos de la Ley 6/1985, de 28 de noviembre, de Ordenación de la Función Pública de la Junta de Andalucía, y se adoptan otras medidas urgentes

Decreto Ley 5/2015, de 15 de septiembre, por el que se modifican el objeto y los fines de las agencias públicas Servicio Andaluz de Empleo y Agencia Pública Andaluza de Educación y Formación, estableciendo el procedimiento para culminar la integración de la Red de Consorcios Escuela de Formal 'ción para el Empleo

Decreto Ley 1/2016, de 15 de marzo, por el que se modifican la Ley 2/2015, de 29 de diciembre, de medidas urgentes para favorecer la inserción laboral, la estabilidad en el empleo, el retorno del talento y el fomento del trabajo autónomo; el Decreto-Ley 6/2014, de 29 de abril, por el que se aprueba el Programa EMPLE@JOVEN y la INICIATIVA@MPRENDE+y el Decreto Ley 9/2014, de 15 de julio, por el que se aprueba el Programa EMPLE@30+

Decreto Ley 4/2016, de 26 de julio, de medidas urgentes relativas al Impuesto sobre Sucesiones y Donaciones

Decreto Ley 5/2016, de 11 de octubre, por el que se regula la jornada de trabajo del personal empleado público de la Junta de Andalucía

Decreto-ley 3/2017, de 19 de diciembre, por el que se regula la Renta Mínima de Inserción Social en Andalucía 
Decreto-ley 1/2018, de 27 de marzo, de medidas para la gestión de los instrumentos financieros del Programa Operativo FEDER Andalucía 20142020 y otras de carácter financiero.

Como puede apreciarse, en relación con el Decreto Ley, esta legislatura deja también un buen bagaje en relación a las posibilidades que ofrece y a la propia expresión de esta fuente del derecho en el ámbito autonómico, después de que dicha figura se incorporara, como es conocido, en la mayoría de las experiencias autonómicas, a raíz de la serie de reformas estatutarias emprendidas a partir de 2006. Cabe llamar la atención, en este sentido, que hasta en dos ocasiones se ha producido la no convalidación por parte de la Cámara del Decreto Ley aprobado por el Consejo de Gobierno, si bien en ambos casos, la introducción de mínimas modificaciones ha permitido una posterior aprobación ${ }^{2}$.

Con respecto a las novedades legislativas más significativas introducidas en esta legislatura, cabe hacer mención igualmente a las políticas legislativas que han prosperado en materia de urbanismo y medio ambiente. Así, de forma significativa destacan:

Ley relativa a modificación de la Ley 6/2016, de 1 de agosto, por la que se modifica la Ley 7/2002, de 17 de diciembre, de ordenación urbanística de Andalucía para incorporar medidas urgentes en relación con las edificaciones construidas sobre parcelaciones urbanísticas en suelo no urbanizable

Ley de Medidas frente al Cambio Climático y para la transición hacia un nuevo modelo energético en Andalucía

Ley de medidas en materia de gestión integrada de calidad ambiental, de aguas, tributaria y de sanidad animal (procedente del Decreto-Ley 3/2015, de 3 de marzo)

Del mismo modo, cabe subrayar la aprobación leyes de especial calado democrático, como lo son la Ley de Participación Ciudadana de Andalucía y la Ley de Memoria Histórica y Democrática de Andalucía; e igualmente, en el ámbito de la comunicación, tanto la Ley del Cine, como la reciente Ley de lo Audiovisual.

Como decíamos al comienzo, la X legislatura bien puede considerarse el punto de partida de una nueva realidad en lo que respecta a la función de control por parte del Parlamento. A este respecto, y más allá de la especial intensidad con la que se

2 Una cuestión a la que nos referimos en "El uso del decreto ley frente a "coyunturas económicas problemáticas" y por gobiernos en minoría", en Administración de Andalucía. Revista Andaluza de Administración Pública, núm. 94 (2016), pp. 249-255 
han puesto en práctica los mecanismos de control en un escenario parlamentario de pluralidad, lo cierto es que esta legislatura nos deja novedades importantes con respecto a lo que es la comprensión de la vida parlamentaria. De entre ellas, tal vez, cabe destacar, en primer lugar, la decisión aprobada por la presidencia del parlamento andaluz, en relación al control del cumplimiento de las mociones ${ }^{3}$, con la cual se pone fin a una laguna evidente en el Reglamento parlamentario que, al contrario del de otros parlamentos autonómicos, nada prevé a este respecto. Una decisión que ha otorgado a los grupos parlamentarios la posibilidad efectiva de controlar el cumplimiento de aquellas mociones que hayan prosperado, pudiendo éstos solicitar un informe público al Consejo de Gobierno en el que este explique el grado de cumplimiento del mismo, debiendo el Presidente de la Cámara situar en el primer punto del orden del día, cuando así se produzca, la ausencia de informe dentro del plazo requerido por parte del Consejo de Gobierno. Cabe señalar aquí que el impulso a la acción de Gobierno por parte del Parlamento ha sido también significativo durante la X legislatura. Concretamente, y en relación con las mociones parlamentarias, han sido aprobadas un total de 27. Y también, un total de 194 proposiciones no de Ley en el Pleno, y 714 en Comisión.

Desde el punto de vista del derecho parlamentario, ha sido igualmente muy significativa la problemática suscitada por la antinomia larvada, hasta ahora irrelevante, entre los artículos 34 y 36 del Reglamento parlamentario, que establecen reglas de para la elección de los miembros de la Mesa del Parlamento potencialmente contradictorias. En concreto, el artículo 34 establece la regla de que los tres vicepresidentes y los secretarios que integran la Mesa del Parlamento se elegirán por orden correlativo a través de una votación en la que los diputados únicamente podrán apoyar con su voto a un candidato de entre los propuestos respectivamente para las tres vicepresidencias y las tres secretarías. Al mismo tiempo, el artículo 36 incorpora desde su reforma de 1996, la regla de que todos los grupos parlamentarios han de estar representados en la mesa. Como es conocido, en la constitución de la Mesa de la X legislatura, el Presidente de edad interpretó, ante la situación de que en aplicación del artículo 34 el último puesto en la Mesa correspondería a una parlamentaria del Partido Popular, y en aplicación del 36 este correspondería al grupo parlamentario de Izquierda Unida, que era la regla del artículo 36 la que había de prevalecer. Como es conocido, la decisión del Presidente de edad dio lugar a un recurso de amparo ante el Tribunal Constitucional por parte de la diputada del Partido Popular ${ }^{4}$, recurso que fue estimado y que provocó una modificación en la composición de la Mesa, integrando a la diputada popular recurrente, que relevaría al diputado de IU que había sido incorporado a la mesa en aplicación de la regla de representación de todos los

\footnotetext{
3 BOPA, núm. 242, de 6 de junio 2016.

$4 \quad$ STC, 199/2016.
} 
grupos que establece el artículo 34. En cualquier caso, la ejecución de esta sentencia no dejaba de presentar aspectos problemáticos fundamentalmente porque la misma implicaba una inaplicación o derogación puntual del artículo 36 del Reglamento. A este respecto, la solución adoptada por el pleno del Parlamento ha sido la de llevar a cabo la única modificación del Reglamento que ha tenido lugar esta legislatura, con la aprobación de la Disposición adicional cuarta, cuyo tenor literal es el siguiente:

Durante la X legislatura del Parlamento de Andalucía, si algún grupo parlamentario no obtuviera ningún representante en la Mesa, deberá contar en ella con un vocal o una vocal, que será nombrado por dicho órgano a propuesta del grupo parlamentario afectado.

El vocal o la vocal, con voz pero sin voto, tendrá derecho de asistencia y opinión en las reuniones de la Mesa; recibirá la información necesaria para el desempeño de sus funciones; colaborará en el normal desarrollo de los trabajos de la Cámara según las disposiciones de la Presidencia; y ejercerá, además, cualesquiera otras funciones que le encomiende esta o la Mesa. No computará a efectos de quórum».

Como vemos, la reforma del Reglamento no ha devuelto, en cualquier caso, la plena eficacia a la previsión del artículo 36, ya que éste precepto no contemplaba una representación mermada sino plena de facultades de todos los grupos parlamentarios en la Mesa del Parlamento, mientras que la solución que para este legislatura se adopta a través de la Disposición adicional cuarta, es la de excluir el voto del representante del grupo parlamentario que no hubiera obtenido su representación ex artículo 34.

De todas las formas, y ahora que nos encontramos ante la inminente elección de la Mesa para la XI legislatura, lo cierto es que hubiera sido necesario una reforma integradora de los artículos 34 y 36 del Reglamento, que diera respuesta a la necesidad de integrar criterios puramente proporcionales para la composición de la Mesa con el lógico interese de garantizar la representación del pluralismo político en ésta, a través de los grupos parlamentarios.

Con respecto a esto último, tal vez cabría cerrar con una reflexión general sobre la cada vez más necesaria reforma del Reglamento del Parlamento andaluz, de cara a suplir carencias y eliminar alguna de sus contradicciones. Más allá de que haya lagunas que impidan el cumplimiento de obligaciones legales y estatutarias, como por ejemplo en lo referido a la rendición de cuentas ante la Cámara de los senadores autonómicos, lo cierto es que optimizar el funcionamiento de ciertas instituciones parlamentarias, requeriría un debate en profundidad sobre la necesidad de reformas de fondo, y no, como hemos visto en el caso de las mociones, soluciones parciales adoptadas por la presidencia. En esta legislatura, sólo el grupo parlamentario de PODEMOS ha planteado una propuesta de reforma del reglamento, pero no 
con carácter general, sino destinada exclusivamente a establecer nuevos mecanismos de cómputo para las dietas y las cesantías, la cual sólo obtuvo el apoyo de Izquierda Unida. Para la nueva legislatura que entra en curso, la reflexión sobre la reforma del derecho parlamentario andaluz seguirá siendo una de las grandes tareas pendientes. 